\title{
UM ALGORITMO GENÉTICO ESPECIALIZADO APLICADO AO PLANEJAMENTO DA EXPANSÃO DO SISTEMA DE TRANSMISSÃO COM ALOCAÇÃO DE DISPOSITIVOS DE COMPENSAÇÃO SÉRIE
}

\author{
Celso Tadao Miasaki* \\ miasaki@mat.feis. unesp.br
}

\author{
Ruben Romero ${ }^{\dagger}$ \\ ruben@dee.feis.unesp.br
}

*Departamento de Matemática - Faculdade de Engenharia de Ilha Solteira - UNESP

Alameda Rio de Janeiro 266, - Telefone: (18) 3743 - 1030

CEP 15385-000, Ilha Solteira, SP, Brasil

${ }^{\dagger}$ Departamento de Engenharia Elétrica - Faculdade de Engenharia de Ilha Solteira - UNESP

Av. Brasil 56, - Telefone: (18) 3743 - 1236

CEP 15385-000, Ilha Solteira, SP, Brasil

\begin{abstract}
This paper proposes a new method for representation of the long-term transmission expansion planning model. The main contribution of this approach is that the developed mathematical model permits to consider the installation of series compensation devices. With the inclusion of these devices, in this new formulation, the objective function becomes non-linear and the behavior of the constraints related to Kirchhoff laws becomes ever more non-linear and complex. In order to validate the proposed model, studies are conducted to verify the active power flow behavior in the presence of series compensation devices. A specialized genetic algorithm, with integer and real codification, was implemented to solve this model. The optimization solution supplies the amount and the local where new transmission lines and series compensation devices must be installed. In the case of series compensation devices, the compensation level capability is also provided. The impacts caused by the installation of the series compen-

\footnotetext{
ARTIGO CONVIDADO:

Versão completa e revisada de artigo apresentado no SBAI-2005

Artigo submetido em 18/06/2006

1a. Revisão em 09/08/2006

2a. Revisão em 09/11/2006

Aceito sob recomendação do Editor Convidado

Prof. Osvaldo Ronald Saavedra Mendez
}

sation devices are presented though detailed studies by using the IEEE 24 bus system. The results show that the method has great potential to be applied to real long-term transmission expansion planning problem.

KEYWORDS: Transmission network expansion planning, series compensation, genetic algorithm.

\section{RESUMO}

Este artigo propõe uma nova estratégia para representar o problema de planejamento da expansão da transmissão a longo prazo. A proposta apresenta uma novidade, um modelo matemático que considera a instalação de dispositivos de compensação série. Nesta nova formulação a inclusão desses dispositivos torna a função objetivo não-linear e aumenta a não-linearidade das restrições relacionadas com a segunda lei de Kirchhoff. Para validar o modelo proposto são estudados o comportamento dos fluxos de potência ativa no sistema com a presença dos dispositivos de compensação série. Um algoritmo genético especializado, com codificação inteira e real, foi implementado com a intenção de fornecer como solução, a quantidade e o local, onde devem ser instalados as novas linhas de transmissão e os dispositivos de compensação série, além do valor da compensação. Os im- 
pactos da adição dos dispositivos de compensação série são mostrados através de estudos detalhados com o sistema teste IEEE 24 barras. Os resultados indicam um bom desempenho da metodologia proposta.

PALAVRAS-CHAVE: Planejamento da expansão de sistemas de transmissão, compensação série, algoritmo genético.

\section{INTRODUÇÃO}

O planejamento do sistema de transmissão começa com o estabelecimento de cenários de crescimento do consumo de energia demandada pela sociedade, de acordo com previsões ao longo do tempo. É a partir desses cenários que se verifica a eventual necessidade de se ampliar e fortalecer a rede. No caso das condições de atendimento não serem satisfeitas, deve-se propor um plano que tenha coerência entre o fornecimento de energia à carga, a demanda e as instalações de novos equipamentos no sistema. Para integrar esses novos equipamentos na rede, de forma a manter as condições técnicas e operacionais adequadas, é preciso planejar a alocação desses reforços sobre o sistema de transmissão. (Soares Neto, 2005)

O principal objetivo do planejamento da expansão do sistema de transmissão é obter o plano ótimo de expansão do sistema elétrico. O melhor plano de expansão deverá indicar onde devem ser alocados novos equipamentos para que o sistema opere de forma adequada, isto é, que obedeça às restrições operacionais e financeiras para um determinado cenário futuro.

A criação de um modelo matemático que represente o problema de planejamento da transmissão começa com algumas hipóteses, onde a exatidão e a complexidade são consideradas na construção do modelo. Geralmente, o problema é representado por um problema não-linear inteiro misto que apresenta um número elevado de soluções ótimas locais para sistemas reais. Este número está associado aos possíveis planos da expansão, que especificam o modo operacional ótimo. Portanto, um problema básico consiste em definir a alternativa de expansão que possua o menor custo e que satisfaça todas as restrições operacionais.

Recentemente, ocorreram mudanças significativas no sistema de transmissão de energia elétrica. Dentre os fatores que contribuiram para que isso ocorresse podem ser mencionadas as restrições de ordem econômica, ambiental, política e geográfica que restringiram os investimentos em unidades de geração e transmissão, necessários para ampliação do sistema. Como consequiência destes obstáculos, têm-se um novo cenário para o sistema elétrico, no qual se observa um aumento das trocas de potência não planejadas. De fato, podem aparecer problemas de fluxo de malha e fluxo paralelo.
Do ponto de vista técnico, esses problemas de fluxo agravam o problema de capacidade de transmissão das linhas, fazendo com que algumas linhas fiquem sobrecarregadas, ao passo que outras ficam com capacidade ociosa de carregamento (Pereira, 2000).

Portanto, existe a necessidade de se adotar novas estratégias para o planejamento do sistema de transmissão, que considerem nos modelos de planejamento a utilização mais eficiente das linhas de transmissão através do controle do fluxo de potência. Assim, os fluxos das linhas sobrecarregadas seriam direcionados para regiões que possuem capacidade ociosa de carregamento, aliviando as regiões de sobrecarga (Taranto et al., 1992).

Os dispositivos de compensação série são adicionados na rede com o intuito de reduzir ou eliminar os problemas de fluxo de malha e fluxo paralelo e, consequentemente, obter uma melhor distribuição dos fluxos de potência ativa. A idéia principal da compensação série está em modificar parte da reatância indutiva da linha com a inclusão de capacitores em série ou de dispositivos que apresentam essas características. Um dispositivo de compensação série tem a capacidade de redirecionar o fluxo de potência ativa através do controle da reatância da linha.

Normalmente são utilizados modelos matemáticos simplificados, modelo DC e de Transportes, para representar o sistema elétrico no problema de planejamento da expansão de sistemas de transmissão. Nesse contexto, o trabalho visa analisar a capacidade do sistema em relação ao transporte de potência ativa. O modelo proposto é uma simplificação da rede e resolve apenas o problema de fluxo de potência ativa. Outros aspectos de análise de desempenho como os problemas de estabilidade, os critérios de segurança e as perdas do sistema, que são tópicos relevantes na expansão da transmissão estão fora do escopo deste trabalho. Os modelos DC e de Transportes consideram somente a adição de linhas de transmissão e transformadores convencionais no problema de planejamento da transmissão.

No contexto atual do mercado de energia, um tema de pesquisa muito relevante é a otimização dos sistemas elétricos. Portanto, é natural a existência de trabalhos voltados para a otimização do problema de planejamento de sistemas de transmissão. Para resolver problemas de otimização complexos que exigem elevado tempo de processamento e apresentam o fenômeno da explosão combinatória podem ser utilizadas as técnicas de otimização chamadas de metaheurísticas, pois elas são especialmente adaptadas para problemas que apresentem um espaço de busca imenso com muitos ótimos locais.

Desta forma, para resolver o problema de otimização combinatória do planejamento de sistemas de transmissão com 
a inclusão da compensação série foi proposto um algoritmo genético especializado. Foram feitas simulações no sistema teste IEEE-24 barras para verificar se os dispositivos de compensação série podem ser modelados no problema de planejamento da expansão da transmissão.

\section{MODELAGEM MATEMÁTICA}

Tendo em vista a importância do problema de planejamento da expansão do sistema de transmissão e da dificuldade em solucioná-lo, faz-se necessário desenvolver, de maneira hábil e eficaz, modelos matemáticos que considerem a utilização mais eficiente das linhas de transmissão através do controle do fluxo de potência.

Normalmente, no planejamento estático da expansão do sistema de transmissão, os componentes do sistema que devem ser modelados e definidos corretamente na formulação matemática são as linhas de transmissão com seus parâmetros e custos, as unidades de geração e de carga, os transformadores e os ângulos das barras. Entretanto, nada impede de que sejam inseridos novos equipamentos. Os modelos DC e de Transportes são exemplos de planejamento estático.

Em um sistema de transmissão, a transferência de potência ativa entre duas barras é inversamente proporcional a reatância indutiva série. Ao compensar a reatância indutiva série em um determinado valor, por meio de um capacitor série, há uma diminuição da impedância elétrica do ramo e existe a possibilidade de aumentar a transferência de potência ativa nesse caminho (ABB, 2002).

A instalação de um dispositivo de compensação série em uma linha de transmissão, faz com que o equipamento se comporte como uma reatância variável. É justamente através do controle dessa reatância na linha que o fluxo de potência ativa pode ser controlado (Verma et al., 2004). Consequentemente, ao instalar dispositivos de compensação série numa linha, sua reatância não pode mais ser considerada uma constante.

Como mencionado anteriormente, foi desenvolvido um modelo matemático, que admite a presença de dispositivos de compensação série e que está baseado no modelo DC. O modelo matemático proposto apresenta a estrutura de um problema de otimização não linear inteiro misto (PNLIM) e, tem como objetivo minimizar o custo de investimento na expansão do sistema.

\subsection{Modelo com Compensação Série}

O modelo matemático modificado para o modelo DC com a inclusão dos dispositivos de compensação série assume a seguinte forma:

$$
\begin{aligned}
\text { Min } v= & \sum_{(i, j)} c_{i j} \cdot n_{i j}+\sum_{(i, j)} h_{i j} \cdot\left(n_{i j}^{0}+n_{i j}\right) \cdot \beta_{i j}+ \\
& +\alpha \sum_{k \in \Gamma} r_{k}
\end{aligned}
$$

sujeito a:

$$
\begin{aligned}
& S \cdot f+g+r=d \\
& f_{i j}-\gamma_{i j}\left(1+\rho_{i j} \cdot \beta_{i j}\right) \cdot\left(n_{i j}^{0}+n_{i j}\right) \cdot\left(\theta_{i}-\theta_{j}\right)=0 \\
& \left|f_{i j}\right| \leq\left(n_{i j}^{0}+n_{i j}\right) \cdot \bar{f}_{i j} \\
& \left|\rho_{i j}\right| \leq \lambda_{i j} \beta_{i j} \\
& 0 \leq n_{i j} \leq \bar{n}_{i j} \\
& 0 \leq g_{k} \leq \bar{g}_{k} \\
& 0 \leq r_{k} \leq d_{k} \\
& n_{i j} \text { Inteiro } \\
& \beta_{i j} \text { Binário } \\
& \theta_{j} \text { Irrestrito } \\
& \rho_{i j} \text { Irrestrito } \\
& f_{i j} \text { Irrestrito }
\end{aligned}
$$

São consideradas constantes do problema:

$c_{i j}$ : custo de uma linha de transmissão adicionada no caminho $i-j$;

$h_{i j}$ : custo de um equipamento que realiza a compensação série;

$\gamma_{i j}$ : susceptância de uma linha no caminho $i-j$;

$n_{i j}^{0}:$ número de circuitos existentes na topologia base;

$\bar{f}_{i j}$ : fluxo máximo permitido de um circuito no caminho $i-$ $j$

$\alpha$ : parâmetro de penalidade devidamente ponderado devido a presença de geração artificial.

$S:$ matriz de incidência nó-ramo transposta do sistema elétrico;

$d:$ vetor de demanda nas barras;

$\bar{n}_{i j}$ : número máximo de circuitos que podem ser adicionados no caminho $i-j$;

$\lambda_{i j}$ : fixa o valor máximo da compensação em 0,3.

São consideradas incógnitas do problema as variáveis: 
$v$ : custo de investimento;

$n_{i j}$ : número de circuitos adicionados no caminho $i-j$;

$\beta_{i j}$ : presença ou ausência de dispositivos de compensação série no caminho $i-j$;

$r_{k}:$ geração fictícia na barra k;

$g$ : o vetor composto de elementos $g_{k}$ (geração na barra k) cujo máximo valor é $\bar{g}$;

$f_{i j}$ : fluxo de potência total no caminho $i-j$;

$\rho_{i j}$ : valor da compensação série no caminho $i-j$;

$\theta_{j}$ : ângulo de fase na barra $\mathbf{j}$;

$f$ : vetor composto de elementos $f_{i j}$;

$r$ : vetor composto de elementos $r_{k}$;

A equação (1) se refere à função objetivo cujas duas primeiras parcelas indicam, respectivamente, o custo de investimento em novas linhas de transmissão e em dispositivos de compensação série. A última parcela se refere ao custo da geração artificial.

O primeiro conjunto de restrições (2) representa as equações correspondentes à lei de Kirchhoff das correntes (LCK), uma equação para cada barra do sistema.

A possibilidade de adicionar dispositivos de compensação série está representada no conjunto de restrições (3), correspondentes à lei de Kirchhoff das tensões (LTK). A presença desse equipamento permite que os fluxos de potência ativa no sistema sejam melhores aproveitados, já que esse equipamento redireciona os fluxos de uma maneira mais efetiva.

As restrições (4) se referem ao limite dos fluxos nas linhas.

Assim como na formulação dos modelos DC e de Transportes, este trabalho considera as linhas de transmissão e os transformadores convencionais da mesma forma e sem diferenciação através das variáveis de decisão $n_{i j}$. Também considera que o dispositivo de compensação pode ser adicionado em série com uma linha de transmissão ou transformador convencional, e pode ser representado como uma variação da susceptância na linha. Ao instalar um dispositivo de compensação série na linha, a susceptância dessa linha passa a ser uma combinação da susceptância original com a susceptância variável do equipamento.

Há um aumento na complexidade do problema de planejamento da transmissão com a inclusão de dispositivos de compensação série quando comparado com os modelos DC e de Transportes, devido a algumas considerações feitas e relacionadas abaixo.
- Há um aumento no número de variáveis do problema, as que indicam a presença do dispositivo de compensação (variável binária) em determinado caminho e as que indicam o valor da compensação (variável irrestrita).

- Os dispositivos operam dentro de uma faixa de compensação, limitados por uma porcentagem do valor da susceptância original da linha. Este fato restringe a região factível, o que dificulta a busca por uma solução.

- O fluxo de potência ativa em um circuito pode ser escrito através da equação $f_{i j}=\gamma_{i j}\left(\theta_{i}-\theta_{j}\right)$ e indica que o fluxo de potência no circuito é diretamente proporcional à susceptância no caminho $i-j$. Percebe-se que com a variação da compensação série existe a possibilidade de aumentar a susceptância da linha e, conseqüentemente, aumentar a capacidade de transferência de potência. Mas, com a adição da variável que representa a compensação série na equação anterior, há também um aumento da não-linearidade devido ao produto da variável de compensação $\rho$ e a variável $\theta$, como conseqüência há um aumento da complexidade na resolução do problema. O conjunto de restrições (3) representa este fato.

- Para identificar propostas de soluções infactíveis, isto é, com corte de carga, foram adicionados geradores artificiais nas barras que possuem cargas. A idéia fundamental é facilitar o processo de resolução do problema da mesma forma como se procede na fase I de um problema de programação linear.

- Um parâmetro $\alpha$ foi utilizado para ajustar as unidades na função objetivo (dólares) e, deve assumir um valor adequado, que torne interessante propostas de solução com pequeno corte de carga. Caso ocorra corte de carga, a função objetivo é penalizada através deste parâmetro $\alpha$ cujo valor mais adequado é igual a 1000000 US/MW obtido de testes experimentais de trabalhos anteriores (Gallego et al. 1998). Neste trabalho esse parâmetro foi considerado fixo. Entretanto, é possível considerálo variável, mas essa variação não interfere de forma significativa no processo de otimização.

- Este trabalho considera a compensação série um dispositivo de expansão do sistema elétrico e como uma variável de controle que pode ser representada como uma variação da reatância do circuito. Entretanto, para representar a compensação série no problema de fluxo de potência, utilizou-se a susceptância (inversa da reatância) do equipamento como uma variável da rede, com uma taxa de compensação $\lambda_{i j}$, sendo seus limites representados através do conjunto de restrições (5).

Com a finalidade de esclarecer a troca sugerida na última consideração, isto é, a mudança da reatância pela susceptân- 
cia no modelo proposto, é apresentado o desenvolvimento das manipulações algébricas considerando uma taxa de compensação $\lambda_{i j}=30 \%$, que foi adotada de acordo com as propostas sugeridas nos trabalhos de (Lie, 1997) e (Gerbex et al., 2001).

$$
x_{k m}^{\text {Nova }}=x_{k m} \pm 0,3 \cdot x_{k m}=(1 \pm 0,3) \cdot x_{k m}
$$

Note que pela expressão (14) pode-se aumentar ou diminuir a reatância de uma linha. Como a susceptância é a inversa da reatância, tem-se:

$$
\begin{array}{r}
\gamma_{k m}^{\text {Nova }}=\frac{1}{(1 \pm 0,3) \cdot x_{k m}}=\frac{1}{x_{k m}} \frac{1}{(1 \pm 0,3)}= \\
=\gamma_{k m} \cdot \frac{1}{(1 \pm 0,3)} \\
\gamma_{k m}^{\text {Nova }}=\left[\frac{1}{1,3} ; \frac{1}{0,7}\right] \cdot \gamma_{k m}=[0,77 ; 1,43] \cdot \gamma_{k m}
\end{array}
$$

Ao analisar os resultados, verifica-se que a nova susceptância irá variar no intervalo de $[0,77 ; 1,43]$ da susceptância original da linha.

Desta forma, na restrição (3), caso o sinal do parâmetro $\rho_{i j}$ seja negativo, haverá uma diminuição da susceptância (aumento da reatância) e tem-se uma compensação indutiva. Por outro lado, caso o sinal de $\rho_{i j}$ seja positivo haverá um aumento da susceptância (diminuição da reatância) e tem-se uma compensação capacitiva.

Aparece de forma explícita no conjunto de restrições (3) a possibilidade de alocar dispositivos de compensação tanto nas linhas existentes na configuração base quanto nas linhas adicionadas no processo de otimização. Assim, o número de dispositivos de compensação série alocados em cada caminho pode ser expresso pela expressão $n c s=\beta_{i j}\left(n_{i j}^{0}+n_{i j}\right)$.

\section{TÉCNICA DE SOLUÇÃO}

Embora o modelo proposto seja um problema de programação não linear inteiro misto (PNLIM) mais complexo que o modelo DC, é possível resolvê-lo através de estratégias utilizadas para resolver o modelo DC, desde que sejam feitos alguns ajustes no processo.

Devido a esta complexidade, nota-se que a maioria das técnicas convencionais de programação linear e não linear, utilizadas para resolver o problema sem a presença de dispositivos de compensação série são inadequados e insuficientes para encontrar uma solução satisfatória para o modelo proposto neste trabalho.

O problema proposto é não convexo e, do ponto de vista teórico, só existe garantia de encontrar soluções sub-ótimas. Então, nesses casos, algoritmos baseados em metaheurísticas podem melhorar a probabilidade para encontrar a solução ótima global. As metaheurísticas possuem flexibilidade, robustez e facilidade de adaptação ao problema de planejamento da expansão da transmissão. A proposta deste trabalho para resolver o problema de planejamento da expansão da transmissão, com a adição de dispositivos de compensação no sistema, é utilizar técnicas metaheurísticas, mais especificamente um algoritmo genético especializado.

\section{ALGORITMO GENÉTICO PROPOSTO}

Os algoritmos genéticos (AG) são adequados para o tratamento de problemas não lineares com variáveis mistas (Soares et al., 2002). Também existem vários livros (Goldberg, 1989) (Michaelewicz, 1994) (Sait e Youssef, 1999) que explicam toda a estrutura e funcionamento de um AG. De acordo com Goldberg é um algoritmo robusto, eficiente e eficaz para vários tipos de problemas.

Dentre as várias pesquisas que aplicam o AG no planejamento da expansão do sistema de transmissão podem ser citados os trabalhos de (Paterni et al., 1999) (Gerbex et al., 2001) (Gallego et al., 1998) e (Da Silva et al., 2000).

A estrutura apresentada pelo modelo matemático proposto faz com que seja necessário alterar algumas etapas do AG tradicional. A seguir são relatadas as principais características do algoritmo genético especializado desenvolvido para o problema de planejamento da transmissão com dispositivos de compensação série.

\subsection{Codifi cação}

No algoritmo genético é necessário codificar uma configuração candidata, isto é, deve-se fazer a representação de uma configuração candidata através de uma cadeia cromossômica.

A codificação utilizada neste trabalho faz referência as linhas de transmissão adicionadas e ao valor compensado na linha.

As linhas de transmissão adicionadas no sistema são representadas por variáveis inteiras indicadas por $n_{i j}$. Já os valores da compensação são codificados por meio de variáveis contínuas e indicadas por $\rho_{i j}$.

Com a intenção de facilitar a compreensão da codificação proposta ilustra-se por meio da figura 1 a topologia de um 


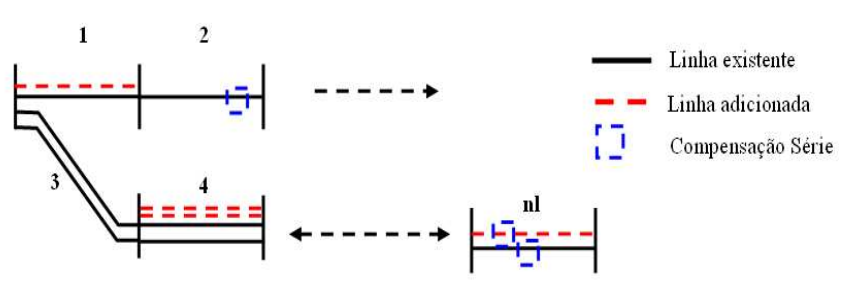

Figura 1: Topologia de um sistema fictício

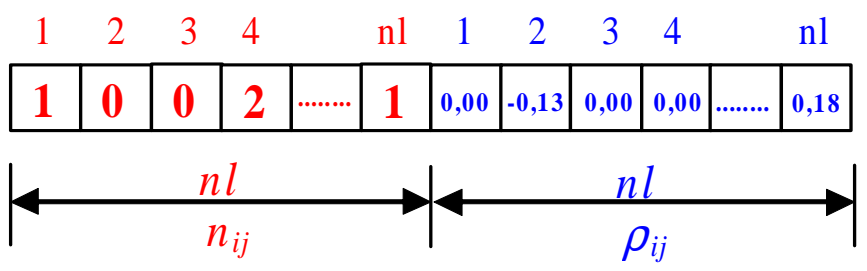

Figura 2: Codificação de uma solução candidata

sistema fictício.

Uma proposta de codificação da topologia desse sistema fictício, para o modelo com compensação série, se encontra ilustrada na figura 2.

$\mathrm{O}$ vetor de tamanho $2 n l$ apresenta duas parcelas distintas, as primeiras $n l$ posições indicam o número de linhas de transmissão adicionadas e as demais posições indicam a porcentagem de compensação em cada caminho, sendo $n l$ o número de caminhos candidatos à adição. Apesar das linhas de transmissão existentes na configuração base não estarem explicitamente codificadas, elas são consideradas no cálculo das variáveis de operação e na adição de compensação série com as linhas de transmissão.

As informações que aparecem na figura 2 são que no caminho 1 é adicionada apenas uma linha de transmissão; no caminho 2 há somente compensação porque existem linhas na configuração base; no caminho 3 não há adição de linhas nem compensação; no caminho 4 houve somente a adição de duas linhas; e no último caminho há adição de uma linha e se faz compensação. Deve-se observar que o valor da compensação pode variar dentro do intervalo permitido e as linhas em paralelo devem ter o mesmo valor de compensação.

Em Gallego (Gallego et al., 1998), um excelente desempenho foi obtido com essa proposta de codificação para o problema de planejamento tradicional (sem compensação série). A codificação implementada também está de acordo com as propostas sugeridas nos algoritmos evolutivos apresentados por Chu (Chu e Beasley, 1997), Da Silva (Da Silva et al., 2000) e Escobar (Escobar et al., 2004).

\subsection{População Inicial}

A escolha da população inicial afeta a qualidade da solução e o número de gerações necessárias para encontrar uma boa solução. Isto ocorre porque as características das soluções candidatas que pertencem a população inicial são transferidas para seus descendentes nas gerações subseqüentes (Sait e Youssef, 1999).

Um mecanismo de controle foi utilizado para gerar os indivíduos da população inicial. O controle se baseia em fixar para cada indivíduo da população: as quantidades máximas de linhas de transmissão adicionadas (10 para cada descendente e 2 em cada caminho) e o número máximo de caminhos com compensação série (3 caminhos).

Neste trabalho é gerada uma população inicial de forma aleatória controlada. Assim, antes de gerar a população inicial, são estudados os fluxos de potência ativa da configuração base, com o intuito de dar preferência às linhas que contém os melhores desempenhos, com relação à quantidade de fluxos transmitidos. Propõe-se um ordenamento dos circuitos baseado em um critério de sensibilidade das linhas que separa os circuitos em dois grupos. Um grupo composto com as linhas melhores classificadas (75\%) a nível de desempenho e outro com os $25 \%$ restantes. Para se calcular o desempenho de todos os circuitos do sistema foi utilizada a seguinte relação:

$$
C o_{i j}=\frac{\bar{f}_{i j} \gamma_{i j}}{c_{i j}} .
$$

Assim, implementa-se a idéia de adicionar linhas de transmissão com melhor desempenho na população inicial. Numa primeira etapa serão escolhidas $80 \%$ das linhas utilizando apenas o maior grupo. O restante das linhas podem ser encolhidas de qualquer grupo. Portanto, a população inicial é composta de 80 a $100 \%$ dos elementos do melhor grupo.

Utilizou-se um processo aleatório, composto de duas etapas. Na primeira etapa, escolhe-se o local e a quantidade de linhas de transmissão a serem adicionadas. A segunda etapa faz referência aos dispositivos de compensação série, na qual são escolhidos o local e o valor compensado.

Com relação a parte da compensação série na população inicial são calculadas dentro de um intervalo de $\pm 30 \%$ do valor da reatância original.

\subsection{Função Adaptação e Infactibilidades}

Para medir a qualidade de um indivíduo da população deve ser proposta uma função de adaptação. No modelo matemático com dispositivos de compensação série a função de adaptação é obtida através da resolução de um problema de 
programação linear (PL). Para cada indivíduo da população corrente é resolvido um PL, que determina o valor exato das variáveis de operação para uma determinada proposta de investimento e verifica se o sistema está operando adequadamente (não apresenta corte de carga). A solução do PL pode indicar uma operação sem corte de carga (adequada) ou pode indicar o valor do corte de carga. Deve-se observar que a única forma eficiente de identificar a qualidade de uma proposta de expansão é verificar o corte de carga obtido ao resolver um problema de PL.

O valor da função de adaptação para cada proposta de solução do modelo proposto é obtido através da relação abaixo. A primeira e segunda parcela indicam, respectivamente, os custos de investimentos em linhas de transmissão e dispositivos de compensação série. A última parcela correspondente ao corte de carga produzido pela configuração em estudo.

$$
v^{k}=\sum_{(i, j) \in \Omega} c_{i j} \cdot n_{i j}^{k}+\sum_{(i, j)} h_{i j} \cdot\left(n_{i j}^{0}+n_{i j}^{k}\right) \cdot \beta_{i j}^{k}+\alpha . w^{k}
$$

Este trabalho considera que o sistema opera de forma adequada quando não existe corte de carga para uma proposta de expansão. Caso uma solução candidata apresente corte de carga, o parâmetro $\alpha$ é utilizado para penalizá-la. Assim, não existe distinção no algoritmo genético especializado, pois todas as propostas de solução são consideradas para participar do processo evolutivo.

Em uma determinada proposta de investimento $k$, após serem identificados os valores das variáveis $n_{i j}^{k}, \rho_{i j}^{k}$ e $\beta_{i j}^{k}$ através do algoritmo genético especializado sugerido, deve-se resolver um PL cuja formulação é a seguinte:

$$
\begin{aligned}
\operatorname{Min} w^{k}= & \sum_{k \in \Gamma} r_{k} \\
& S \cdot f+g+r=d \\
& f_{i j}-\gamma_{i j}\left(1+\rho_{i j}^{k} \cdot \beta_{i j}^{k}\right)\left(n_{i j}^{0}+n_{i j}^{k}\right) . \\
& \cdot\left(\theta_{i}-\theta_{j}\right)=0 \\
& \left|f_{i j}\right| \leq\left(n_{i j}^{0}+n_{i j}^{k}\right) \cdot \bar{f}_{i j} \\
& 0 \leq g \leq \bar{g} \\
& 0 \leq r \leq d \\
& f_{i j} \text { Irrestrito } \\
& \theta_{j} \text { Irrestrito }
\end{aligned}
$$

\subsection{Seleção}

A seleção é o operador genético que permite comparar e selecionar dentro da população corrente as soluções candidatas que participarão da formação da nova geração. O processo de seleção tem como objetivo destacar os melhores indivíduos de uma população na esperança de obter descendentes mais bem adaptados que seus antecessores.

Neste trabalho foi empregada a seleção baseada em torneio, sendo gerada aleatoriamente uma sub-população composta de 2 indivíduos. Dentre eles é escolhido o indivíduo com melhor função de adaptação. Este processo é repetido até que a população esteja completa.

A seleção por torneio é uma das formas mais utilizadas devido a sua eficiência e simplicidade na implementação de problemas de maximização ou minimização. Uma vantagem da seleção por torneio em relação à seleção proporcional está em não ser necessário integralizar as variáveis, e também porque os valores absolutos das funções objetivos não são tão significativos.

\subsection{Recombinação}

Após o processo de seleção é implementado o mecanismo de recombinação. Implementou-se neste trabalho a recombinação com um ponto de corte, na qual são escolhidas duas soluções candidatas para gerar dois descendentes. O processo se inicia com a escolha aleatória de um ponto de recombinação que irá gerar seus descendentes, com uma parcela de cada uma das topologias geradoras separadas pelo ponto de recombinação.

No algoritmo implementado, apesar do vetor de codificação ter tamanho $2 n l$, o ponto de recombinação foi escolhido por meio de um número aleatório gerado entre 1 e $(n l-1)$. O motivo é que este modelo considera apenas a primeira metade do vetor de codificação. Assim, na recombinação, a segunda parcela do vetor de codificação acompanha a lógica da primeira parcela conforme demonstrada na figura 3 .

A estratégia fundamental consiste em repassar a informação existente em cada caminho (presente nos dois setores do vetor de codificação) para um único descendente e, desta forma, evitar a degradação da qualidade da informação existente no vetor de codificação.

\subsection{Mutação}

O algoritmo genético especializado proposto apresenta dois tipos de mutação que são designados por mutação1 e mutação2. A escolha do tipo de mutação está relacionada diretamente com uma taxa de diversificação da população corrente, cuja fórmula será apresentada mais adiante. Caso a taxa de diversificação fique acima de $40 \%$ é feita a mutação1. Caso contrário, é realizada a mutação2.

Na mutação1, para cada indivíduo da população corrente é resolvido um problema subsidiário, no caso, um problema de programação linear. Posteriormente, as linhas são divi- 

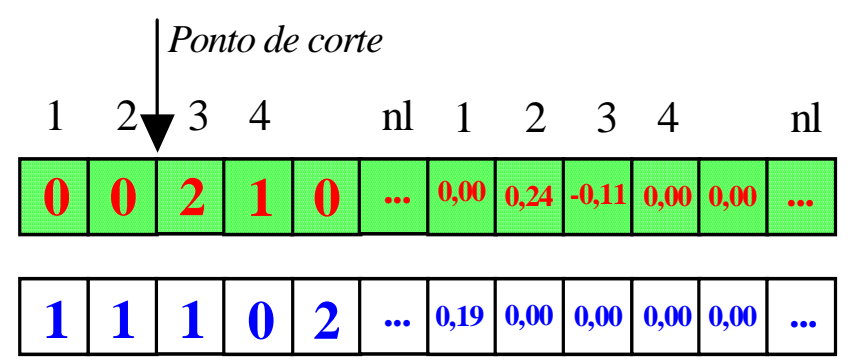

(a) Pais Escolhidos
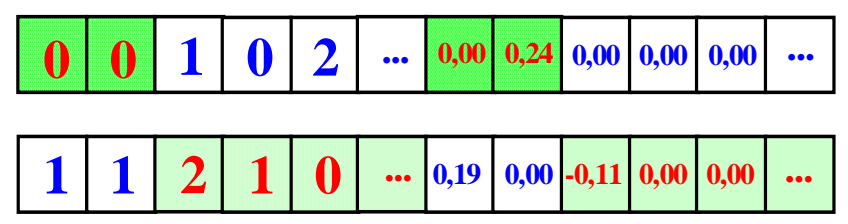

(b) Descendentes Gerados

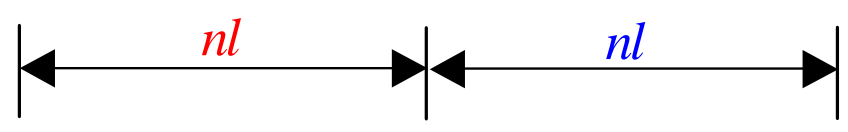

Figura 3: Processo de Recombinação (a) antes da Recombinação (b) depois da Recombinação

didas em quatro grupos de acordo com um índice de aproveitamento. Isto foi feito com a intenção de dar preferência a retirada de linhas com baixo índice de aproveitamento e a colocação de linhas nos caminhos com índices elevados. O índice de aproveitamento da linha é dado pela fórmula:

$$
C f_{i j}=\frac{f_{i j}}{\left(n_{i j}^{0}+n_{i j}\right) \cdot \bar{f}_{i j}}
$$

Com a idéia de diminuir os custos de investimentos de uma solução candidata, a operação de mutação1 aplica um processo probabilístico com a seguinte ordem de prioridade: 1) a retirada de linhas e/ou dispositivos de compensação série; 2) colocação de dispositivos de compensação série e troca do valor compensado; 3) Colocação de linhas (com menor chance de ocorrer).

O motivo desta estratégia é inverter a tendência de adicionar linhas porque a maioria dos elementos do vetor de codificação se encontra com valor igual a zero, o que neste caso aumentaria o custo de investimento. Também deve-se salientar que esta mutação é realizada em apenas um ponto.

A mutação1 depende da parcela escolhida no cromossomo. Caso seja escolhido um caminho da parcela correspondente às linhas de transmissão então, deve-se aumentar ou diminuir uma linha de transmissão nesse caminho $\left(n_{i j}+1\right.$ ou $\left.n_{i j}-1\right)$. Caso seja escolhido um caminho da parcela correspondente aos dispositivos de compensação, deve-se adicionar ou retirar compensadores no caminho escolhido.

A mutação2 é realizada quando a taxa de diversificação fica abaixo de $40 \%$, e apenas com os descendentes que possuem uma ou mais configurações idênticas na população corrente. A mutação2 é feita em múltiplos pontos, mas não superior a $20 \%$ dos caminhos da configuração em estudo.

No intuito de obter eficiência, os dois processos de mutação propostos obedecem a alguns critérios fundamentais para que não haja produção de adições contraditórias como a inclusão de dispositivos de compensação série num caminho onde não existem linhas. Também são levados em conta na mutação que quando for adicionado ao sistema:

- O número de dispositivos de compensação série deve ser igual ao número de linhas de transmissão nesse caminho;

- Deve-se respeitar o número máximo de linhas de transmissão a ser adicionado em cada caminho;

- Caso seja retirada uma linha de transmissão, deve-se retirar também, caso exista, o dispositivo de compensação série correspondente.

Portanto, a mutação não deve produzir adições incoerentes, isto é, a inclusão de dispositivos de compensação em um caminho está condicionada a existência de linhas e o número de dispositivos de compensação deve ser igual ao número de linhas de transmissão nesse caminho.

Para ilustrar o processo de mutação, são apresentados dois casos na figura 4.

O ponto de mutação é escolhido aleatoriamente. No primeiro caso foi escolhido o caminho 3 que se refere a compensação série. Embora não ocorra adição de linhas, há um determinado número de linhas na configuração base e, portanto, o número de dispositivos de compensação série instalado deve ser igual ao número de linhas existentes na configuração base.

No segundo caso foi escolhido o caminho 1 que faz menção a retirada da linha de transmissão. Supondo que não exista linha na configuração base, a retirada desta única linha faz com que seja necessário retirar também a compensação série no caminho 1 para manter a coerência do processo. 
Ponto de mutação:

\begin{tabular}{|c|c|c|c|c|c|c|c|c|c|c|c|}
\hline 1 & 2 & 3 & 4 & & $\mathrm{nl}$ & 1 & 2 & 3 & \multicolumn{2}{|l|}{4} & $\mathrm{nl}$ \\
\hline 0 & 2 & 0 & 1 & 2 & ... & 0,00 & 0,24 & 0,00 & 0,00 & 0,00 & ... \\
\hline 1 & 0 & 1 & 2 & 0 & ... & 0,19 & 0,00 & 0,11 & 0,00 & 0,00 & $\ldots$ \\
\hline
\end{tabular}

(a) Pais Escolhidos

\begin{tabular}{|l|l|l|l|l|l|l|l|l|l|l|l|}
\hline 0 & 2 & 0 & 1 & 2 & $\ldots$ & 0,00 & 0,24 & $-0,27$ & 0,00 & 0,00 & $\ldots$ \\
\hline
\end{tabular}

\begin{tabular}{|l|l|l|l|l|l|l|l|l|l|l|l|}
\hline $\mathbf{0}$ & $\mathbf{0}$ & 1 & 2 & $\mathbf{0}$ & $\ldots$ & 0,00 & 0,00 & 0,11 & 0,00 & 0,00 & $\ldots$ \\
\hline
\end{tabular}

(b) Descendentes Gerados

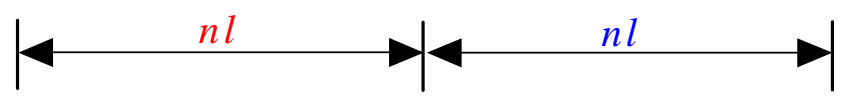

Figura 4: Processo de Mutação

(a) antes da mutação (b) depois da mutação

\subsection{Detalhes Específicos do Algoritmo Genético Proposto}

As características relatadas abaixo fazem referência a um conjunto de parâmetros que contribuem para um melhor desempenho do algoritmo genético proposto.

\subsubsection{Parâmetros de Controle}

Os parâmetros de controle utilizados nos testes foram uma taxa de recombinação de 0,8 , uma taxa de 0,1 para a mutação1 e 0,6 para a mutação 2 e, tamanho da população entre 40 e 150 indivíduos.

\subsubsection{Elitismo}

Com a finalidade de que as melhores topologias da população corrente sejam preservadas e repassadas para a próxima geração foi utilizado um mecanismo de elitismo. O processo de elitismo repassa para a próxima geração os $30 \%$ melhores indivíduos da população corrente.

\subsubsection{Critério de Parada}

O algoritmo termina quando for atingido um número máximo de iterações previamente especificado, que neste estudo foi de 500 gerações.

\subsubsection{Diversidade Populacional}

O mecanismo de controle da diversidade da população está diretamente relacionado com o processo de mutação. O objetivo da diversificação é produzir novos indivíduos na expectativa de encontrar melhores soluções.

Em uma determinada população, a taxa de diversificação pode ser obtida através da fórmula:

$$
T_{\text {Div }}=100\left(1,0-\frac{\text { número de elementos repetidos }}{\text { número total de elementos }}\right)
$$

\subsection{Estrutura do Algoritmo Genético}

A estrutura básica do algoritmo genético especializado é a seguinte:

1. Leitura dos dados de entrada e especificação dos parâmetros de controle;

2. Gerar a população inicial que se transforma na população corrente;

3. Calcular a função de adaptação de todos os indivíduos da população corrente;

4. Calcular a taxa de diversificação. Se for possível, atualizar a incumbente;

5. Implementar a seleção por torneio com $\mathrm{k}=2$;

6. Implementar o elitismo;

7. Implementar a recombinação de um ponto;

8. Implementar a mutação1 ou mutação2;

9. Encontrar a função de adaptação de todos os indivíduos da população corrente, resolvendo um PL para cada elemento da população;

10. Calcular a taxa de diversificação. Se for possível, atualizar a incumbente;

11. Recompor a população corrente;

12. Se o critério de parada for satisfeito pare o processo. Em caso contrário ir ao passo 5.

\section{TESTES COM O ALGORITMO PRO- POSTO}

A partir do modelo matemático com dispositivo de compensação proposto para resolver o problema de planejamento da 
expansão do sistema de transmissão de energia elétrica, foi desenvolvido e implementado um programa computacional para a solução desse modelo.

O sistema teste utilizado para ilustrar os estudos desenvolvidos neste trabalho foi o sistema IEEE 24 barras, que corresponde ao planejamento com reprogramação da geração. Este sistema é composto de 24 barras e 41 caminhos, com demanda de 8.550 MW e capacidade de geração de 10.215 MW. Os demais dados referentes a este sistema se encontram em Fang e Hill (Fang e Hill, 2003) e no apêndice A.

O computador utilizado foi um Pentium IV, $996 \mathrm{MHz}$ com 512Mbytes de memória RAM, plataforma Windows XP. O programa computacional implementado foi elaborado na linguagem de programação Fortran. Além disso, todos os problemas de programação linear deste trabalho foram resolvidos por meio de uma sub-rotina comercial de PL, em linguagem Fortran desenvolvido pela Universidade de Stanford, que faz parte do software conhecido como MINOS (Modular Incore Nonlinear Optimization Systems), versão 5.4.

No processo de otimização são encontrados os valores de geração (real e fictícia) nas barras, os ângulos das barras e os fluxos nas linhas.

Adotou-se o custo da geração artificial $\alpha$ de um milhão de dólares por MW ( $\left.10^{6} \mathrm{US} / \mathrm{MW}\right)$. A estratégia de escolher esse valor, para o custo da geração artificial, foi feita com a intenção de tornar mais atrativo a utilização dos dispositivos de compensação e a adição de linhas de transmissão, encontrando planos de expansão sem corte de carga.

Neste trabalho foi fixado o valor do dispositivo de compensação série em 2 milhões de dólares. Deve-se salientar que este valor pode não corresponder à realidade porque se trata de dispositivos com custos que variam muito. Assim, a proposta apresentada tem interesse acadêmico e para aplicação prática, deve-se procurar os valores exatos desse tipo de dispositivos.

Na tabela 1 são apresentados os resultados obtidos ao aplicar o sistema teste para o modelo DC tradicional, isto é, sem considerar a compensação série como elemento de expansão e para o modelo com compensação série.

As linhas de transmissão são adicionados nos mesmos circuitos que o modelo DC tradicional, com a diferença que foi retirada 1 linha no caminho 07-08 e substituída por dispositivos de compensação série nos caminhos 03-24 e 10-11.

O custo de investimento do modelo proposto é igual a 140 milhões de dólares, uma diferença de 12 milhões em relação a configuração encontrada com o modelo DC.

Foram resolvidos 26094 PL's, que serviram de base para ava-
Tabela 1: Resultados Obtidos Com os Modelos DC e com Compensação Série.

\begin{tabular}{|c||c||c||c|}
\hline & \multicolumn{1}{|c||}{$\begin{array}{c}\text { Modelo } \\
\text { DC }\end{array}$} & \multicolumn{2}{c|}{ Modelo Proposto } \\
\hline Circuito & $\begin{array}{c}\text { Linhas adi- } \\
\text { cionadas }\end{array}$ & $\begin{array}{c}\text { Linhas adi- } \\
\text { cionadas }\end{array}$ & $\begin{array}{c}\text { Valor } \\
\text { Compen- } \\
\text { sado } \\
\rho_{i j}\end{array}$ \\
\hline$n_{06-10}$ & 1 & 1 & - \\
\hline$n_{07-08}$ & 2 & 1 & - \\
\hline$n_{10-12}$ & 1 & 1 & - \\
\hline$n_{14-16}$ & 1 & 1 & - \\
\hline vcs $03-24$ & & & $-0,2662$ \\
\hline vcs $10-11$ & & 136 & 0,1218 \\
\hline $\begin{array}{c}\text { CUSTO } \\
\text { PARCIAL }\end{array}$ & 152 & & 4 \\
\hline $\begin{array}{c}\text { CUSTO } \\
\text { TOTAL }\end{array}$ & 152 & \multicolumn{2}{c|}{} \\
\hline \multicolumn{2}{|c||}{140} \\
\hline
\end{tabular}

liar o esforço computacional do algoritmo genético proposto.

\section{CONCLUSÕES}

O presente trabalho descreveu o desenvolvimento de uma nova metodologia para o problema de planejamento da transmissão, cuja relevância se encontra na possibilidade de considerar os dispositivos de compensação série como componentes da expansão de um sistema elétrico.

O modelo matemático proposto, que aloca linhas de transmissão e dispositivos de compensação série, pode ser utilizado para resolver o problema de planejamento da expansão do sistema de transmissão.

No modelo matemático que representa o problema de planejamento da transmissão, a instalação de dispositivos de compensação série no sistema afeta as equações do fluxo de carga, fazendo com que ocorra um aumento da nãolinearidade do modelo e, conseqüentemente, de sua complexidade.

As simulações mostraram que a proposta de alocar dispositivos de compensação série é viável tecnicamente e bastante atrativa, já que os dispositivos podem reduzir os custos de investimento em linhas de transmissão na expansão da rede.

O algoritmo genético especializado proposto mostrou-se muito eficiente para encontrar a alocação das linhas de transmissão e dos dispositivos de compensação série, pois convergiu para soluções de boa qualidade. 
Os casos estudados com o sistema IEEE 24 barras confirmam que o modelo proposto e o algoritmo desenvolvido podem ser utilizados no planejamento do sistema, já que se mostraram eficazes e eficientes.

\section{AGRADECIMENTOS}

Este trabalho foi realizado com o apoio financeiro da CAPES. Os autores agradecem ao editor associado e aos revisores por seus comentários e sugestões.

\section{REFERÊNCIAS}

ABB Group (2002) Overview about FACTS Applying to your Electric Power System, Disponível em: <http://www.abb.com/facts> Acesso em 25/05/2002.

Chu, P. C.; Beasley, J. E. (1997) An Genetic Algorithm for the Generalized Assignment Problem, Computers Operations Research, vol. 24, $\mathrm{n}^{\circ}$ 1, pp. 17-23.

Da Silva, E.L. Gil, H.A. Areiza, J.M. (2000). Transmission network expansion planning under an improved genetic algorithm, IEEE Transactions on Power Systems, Vol.15, pp. 1168-1175.

Escobar, A.; Gallego, R. A.; Romero, R. (2004) Multistage and Coordinated Planning of the Expansion of the Transmission Systems, IEEE Transactions on Power Systems, vol. 19, ${ }^{\circ}$ 2, pp. 735-744.

Fang, R. Hill, D. (2003). A New Strategy for Transmission Expansion in Competitive Electricity Markets, IEEE Transactions on Power Systems, Vol.18, pp. 374-380

Gallego, R. Monticelli, A. Romero, R. (1998). Transmission system expansion planning by extended genetic algorithm, IEE Proceedings - Generation, Transmission and Distribution, Vol 145, pp. 329-335.

Gerbex, S. Cherkaoui, R. Germond, J. (2001). Optimal Location of Multi-Type FACS devices in Power System by Means of Genetic Algorithms, IEEE Transactions on Power Systems, Vol 16, pp.537-544.

Goldberg, D. (1989). Genetic Algorithms in Search Optimization, and Machine Learning, Addison-Wesley Reading, Mass.

Lie, T.T. Deng, W. (1997). Optimal flexible AC transmission systems (FACTS) devices allocation, Electrical power \& Energy Systems, Vol. 19, $\mathrm{n}^{\circ} 2$, pp. 125-134. .

Michalewicz, Z. (1994). Genetic Algorithm + Data Structures $=$ Evolution Programs, Artificial Intelligence, Springer-Verlag.
Paterni, P. Vitet, S. Bena, M. Yokoyama, Y. (1999) Optimal Location of Phase Shifters in French Network by Genetic Algorithm, IEEE Transactions on Power Systems, vol. 14 , pp. 37-42.

Pereira, J. L. R. (2000). Modelagem de Dispositivos de Controle FACTS em sistemas de Potência para Análise em Regime Permanente, Minicurso do XIII Congresso Brasileiro de Automática.

Sait, S. M. Youssef, H. (1999). Iterative Computer Algorithms with Applications in Engineering: Solving Combinatorial Optimization Problems, IEEE Computer Society Press, CA, Los Amitos.

Soares, L. A. E. Saldanha, R. R. Takahashi, R. H. C. (2002). Algoritmo genético multiobjetivo para otimização de sistemas de distribuição de energia, XIV Congresso Brasileiro de Automática.

Soares Neto, J. B. G. (2005) Planejamento Soma Competência em Novos Projetos, Revista Furnas. Disponível em: <http://www.furnas.com.br/arqtrab/ddppg/revistaonline /linhadireta/rf319_entrev.pdf >. Acesso em 11 jul. 2005.

Taranto, G. N. Pinto, L.M.V.G. Pereira, M.V.F. (1992). Representation of FACTS devices in power system economic dispatch, IEEE Transactions on Power Systems, Vol.7, pp. 572-576.

Verma, A. K.; Srividya, A.; Deka, C. (2004) Impact of a FACTS Controller on Reliability of Composite Power Generation and Transmission System, Electric Power Systems Research, Vol. 72, pp. 125-130.

\section{Apêndice A}

Este apêndice mostra a configuração base e os dados do sistema teste IEEE 24 barras utilizado para resolver o problema de planejamento da expansão do sistema de transmissão. 


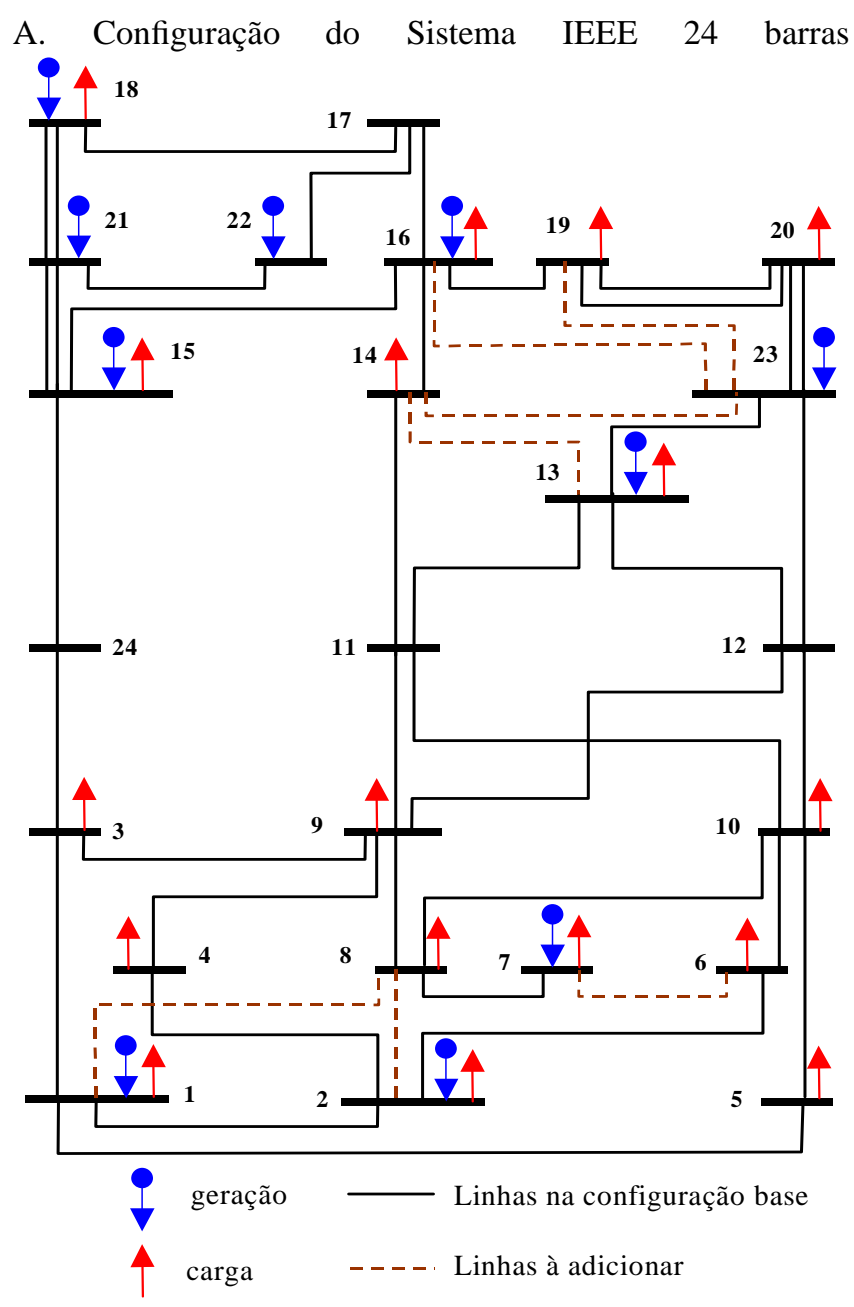

B. Dados das Barras

\begin{tabular}{|c|c|c|}
\hline BARRA & Geração (MW) & Carga (MW) \\
\hline 1 & 576 & 324 \\
\hline 2 & 576 & 291 \\
\hline 3 & 0 & 540 \\
\hline 4 & 0 & 222 \\
\hline 5 & 0 & 213 \\
\hline 6 & 0 & 408 \\
\hline 7 & 900 & 375 \\
\hline 8 & 0 & 513 \\
\hline 9 & 0 & 525 \\
\hline 10 & 0 & 585 \\
\hline 11 & 0 & 0 \\
\hline 12 & 0 & 0 \\
\hline 13 & 1773 & 795 \\
\hline 14 & 0 & 582 \\
\hline 15 & 645 & 951 \\
\hline 16 & 465 & 300 \\
\hline 17 & 0 & 0 \\
\hline 18 & 1200 & 999 \\
\hline 19 & 0 & 543 \\
\hline 20 & 0 & 384 \\
\hline 21 & 1200 & 0 \\
\hline 22 & 900 & 0 \\
\hline 23 & 1980 & 0 \\
\hline 24 & 0 & 0 \\
\hline
\end{tabular}


C. Dados das Linhas

\begin{tabular}{|c|c|c|c|c|c|}
\hline $\begin{array}{l}\mathbf{N}^{o} \text { da } \\
\text { Linha }\end{array}$ & Circuito & $\begin{array}{l}\text { Linhas } \\
\text { existentes }\end{array}$ & Reatância & $\begin{array}{c}\text { Capacidade } \\
\text { transmissão } \\
(M W)\end{array}$ & $\begin{array}{c}\text { Custo } \\
\text { US\$10 }\end{array}$ \\
\hline 1 & $1-2$ & 1 & 0,0139 & 175 & 3000 \\
\hline 2 & $1-3$ & 1 & 0,2112 & 175 & 55000 \\
\hline 3 & $1-5$ & 1 & 0,0845 & 175 & 22000 \\
\hline 4 & $2-4$ & 1 & 0,1267 & 175 & 33000 \\
\hline 5 & $2-6$ & 1 & 0,1920 & 175 & 50000 \\
\hline 6 & $3-9$ & 1 & 0,1190 & 175 & 31000 \\
\hline 7 & $3-24$ & 1 & 0,0839 & 400 & 50000 \\
\hline 8 & $4-9$ & 1 & 0,1037 & 175 & 27000 \\
\hline 9 & $5-10$ & 1 & 0,0883 & 175 & 23000 \\
\hline 10 & $6-10$ & 1 & 0,0605 & 175 & 16000 \\
\hline 11 & $7-8$ & 1 & 0,0614 & 175 & 16000 \\
\hline 12 & $8-9$ & 1 & 0,1651 & 175 & 43000 \\
\hline 13 & $8-10$ & 1 & 0,1651 & 175 & 43000 \\
\hline 14 & $9-11$ & 1 & 0,0839 & 400 & 50000 \\
\hline 15 & $9-12$ & 1 & 0,0839 & 400 & 50000 \\
\hline 16 & $10-11$ & 1 & 0,0839 & 400 & 50000 \\
\hline 17 & $10-12$ & 1 & 0,0839 & 400 & 50000 \\
\hline 18 & $11-13$ & 1 & 0,0476 & 500 & 66000 \\
\hline 19 & $11-14$ & 1 & 0,0418 & 500 & 58000 \\
\hline 20 & $12-13$ & 1 & 0,0476 & 500 & 66000 \\
\hline 21 & $12-23$ & 1 & 0,0966 & 500 & 134000 \\
\hline 22 & $13-23$ & 1 & 0,0865 & 500 & 120000 \\
\hline 23 & $14-16$ & 1 & 0,0389 & 500 & 54000 \\
\hline 24 & $15-16$ & 1 & 0,0173 & 500 & 24000 \\
\hline 25 & $15-21$ & 2 & 0,0490 & 500 & 68000 \\
\hline 26 & $15-24$ & 1 & 0,0519 & 500 & 72000 \\
\hline 27 & $16-17$ & 1 & 0,0259 & 500 & 36000 \\
\hline 28 & 16-19 & 1 & 0,0231 & 500 & 32000 \\
\hline 29 & $17-18$ & 1 & 0,0144 & 500 & 20000 \\
\hline 30 & $17-22$ & 1 & 0,1053 & 500 & 146000 \\
\hline 31 & $18-21$ & 2 & 0,0259 & 500 & 36000 \\
\hline 32 & $19-20$ & 2 & 0,0396 & 500 & 55000 \\
\hline 33 & $20-23$ & 2 & 0,0216 & 500 & 30000 \\
\hline 34 & $21-22$ & 1 & 0,0678 & 500 & 94000 \\
\hline 35 & $1-8$ & 0 & 0,1344 & 175 & 35000 \\
\hline 36 & $2-8$ & 0 & 0,1267 & 175 & 33000 \\
\hline 37 & $6-7$ & 0 & 0,1920 & 175 & 50000 \\
\hline 38 & $13-14$ & 0 & 0,0447 & 500 & 62000 \\
\hline 39 & $14-23$ & 0 & 0,0620 & 500 & 86000 \\
\hline 40 & $16-23$ & 0 & 0,0822 & 500 & 114000 \\
\hline 41 & $19-23$ & 0 & 0,0606 & 500 & 84000 \\
\hline
\end{tabular}

